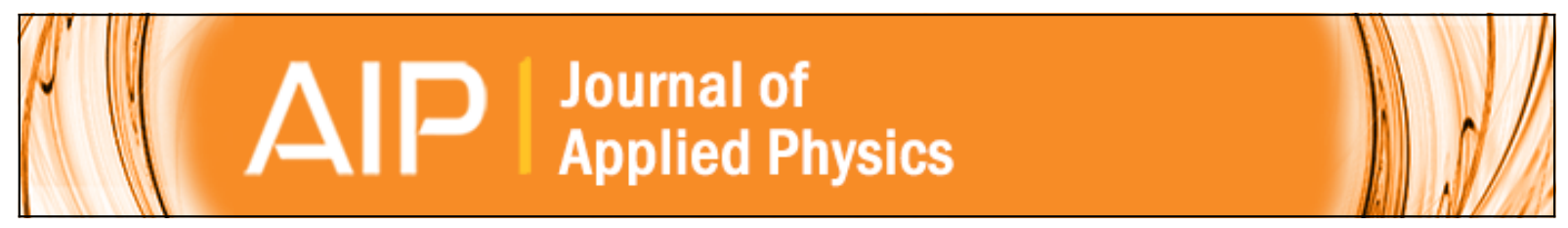

\title{
Off-axis electron holographic potential mapping across AIGaAs/AIAs/GaAs heterostructures
}

Suk Chung, Shane R. Johnson, Yong-Hang Zhang, David J. Smith, and Martha R. McCartney

Citation: Journal of Applied Physics 105, 014910 (2009); doi: 10.1063/1.3062449

View online: http://dx.doi.org/10.1063/1.3062449

View Table of Contents: http://scitation.aip.org/content/aip/journal/jap/105/1?ver=pdfcov

Published by the AIP Publishing

\section{Articles you may be interested in}

Quantitative dopant profiling of $p$ - $n$ junction in In Ga As/Al Ga As light-emitting diode using off-axis electron holography

J. Vac. Sci. Technol. B 28, C1D11 (2010); 10.1116/1.3244575

Polarization field mapping of Al 0.85 In 0.15 N / AIN / GaN heterostructure

Appl. Phys. Lett. 94, 121909 (2009); 10.1063/1.3108084

Mapping the electrostatic potential across $\mathrm{Al} \mathrm{Ga} \mathrm{N/Al} \mathrm{N/Ga} \mathrm{N} \mathrm{heterostructures} \mathrm{using} \mathrm{electron} \mathrm{holography}$ Appl. Phys. Lett. 90, 032101 (2007); 10.1063/1.2431716

Mapping of dopant concentration in a GaAs semiconductor by off-axis phase-shifting electron holography Appl. Phys. Lett. 89, 244101 (2006); 10.1063/1.2402907

Mapping electrostatic potential across an AIGaN/InGaN/AIGaN diode by electron holography Appl. Phys. Lett. 76, 3055 (2000); 10.1063/1.126577

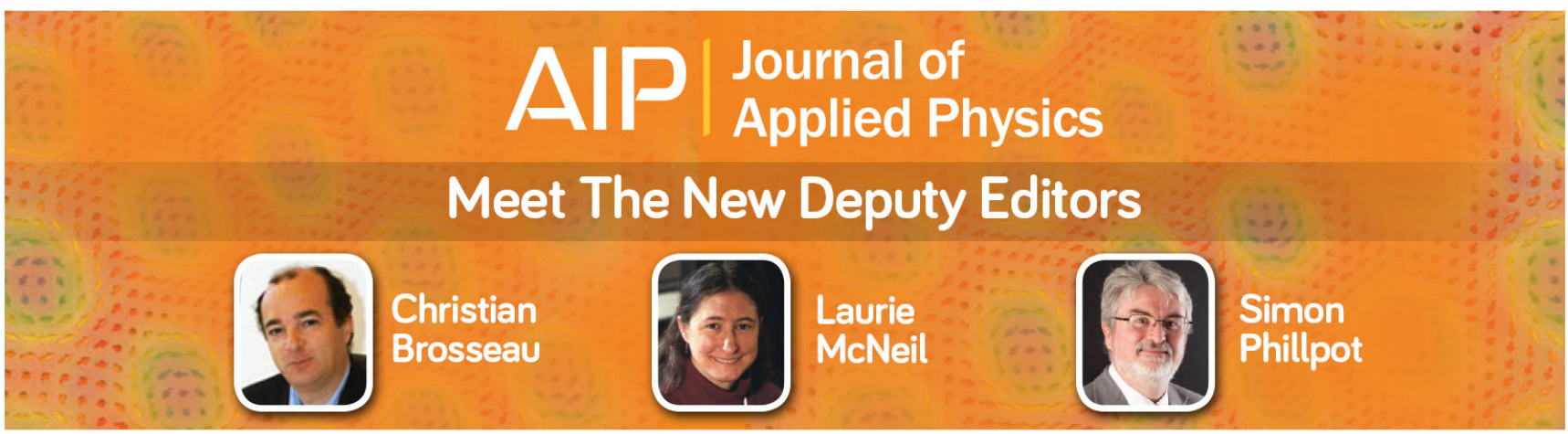




\title{
Off-axis electron holographic potential mapping across AIGaAs/AIAs/GaAs heterostructures
}

\author{
Suk Chung, ${ }^{1, a)}$ Shane R. Johnson, ${ }^{2}$ Yong-Hang Zhang, ${ }^{2}$ David J. Smith, ${ }^{3}$ and \\ Martha R. McCartney ${ }^{3}$ \\ ${ }^{1}$ School of Materials, Arizona State University, Tempe, Arizona 85287, USA \\ ${ }^{2}$ Department of Electrical Engineering, Arizona State University, Tempe, Arizona 85287, USA \\ ${ }^{3}$ Department of Physics, Arizona State University, Tempe, Arizona 85287, USA
}

(Received 24 September 2008; accepted 20 November 2008; published online 13 January 2009)

\begin{abstract}
The electrostatic potential profile across AlGaAs/AlAs/GaAs heterostructures containing 1 - $\mu$ m-thick $n$-doped (or $p$-doped) AlGaAs layers is measured using off-axis electron holography. Simulations of the potential profiles assuming no unintentional impurities in the undoped regions of the samples show small discrepancies with experiment. Revised simulations reproduce the measurements accurately, when a $p$-layer with an $8.4 \times 10^{11} \mathrm{~cm}^{-2}$ acceptor density is included at the buffer/substrate interface to simulate the presence of unintentional carbon impurities. (C) 2009 American Institute of Physics. [DOI: 10.1063/1.3062449]
\end{abstract}

\section{INTRODUCTION}

$\mathrm{AlGaAs} / \mathrm{GaAs}$ heterostructures have attracted intense interest for both fundamental studies in solid state physics and optoelectronic applications, such as laser diodes, lightemitting diodes, and high electron mobility transistors, ever since Esaki and Tsu ${ }^{1,2}$ suggested the possible novel properties of these heterostructures. However, impurities located at the epilayer/substrate interface have often been reported to degrade the expected performance of GaAs heterostructure devices. ${ }^{3,4}$ Studies to determine the nature of these interfacial impurities have been conducted using a range of techniques including photoreflectance spectroscopy, ${ }^{5,6}$ capacitanceversus-voltage measurements, ${ }^{7}$ deep-level transient spectroscopy, ${ }^{8}$ and secondary ion mass spectroscopy. ${ }^{9,10}$ According to these studies, it has been shown that the presence of interfacial carbon impurities shifts the threshold voltage of field-effect transistors due to changes in band structure, ${ }^{3}$ which reduces the electron mobility and density in the channel as the thickness of the buffer layer between the substrate and active channel decreases. ${ }^{10}$ It has also been found that p-type conducting interfacial layers of carbon at the epilayer/ substrate interface cause side gating. ${ }^{4}$

As the dimensions of semiconductor devices decrease, determining the electrostatic potential distribution within deep-submicron heterostructures represents an imposing challenge. Electron holography in the electron microscope is an interferometric technique that can measure the phase change in an electron wave passing through a specimen with nanometer-scale resolution. ${ }^{11}$ Since this phase change depends on the local electrostatic potential, the technique has proven to be a powerful tool for mapping out potential variations caused by charges such as activated dopants. ${ }^{12}$ In this research, off-axis electron holography is used to determine the electrostatic potential profile across AlGaAs/AlAs/GaAs heterojunctions. The electrostatic potential drop at the

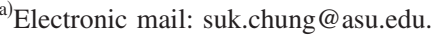

epilayer/substrate interface and the corresponding sheet concentrations of charge due to interfacial impurities are determined and compared with previous studies.

\section{EXPERIMENTAL DETAILS}

Two different samples grown by molecular beam epitaxy in a VG V80H solid-source system were studied. The first sample was $n$-doped and consisted of a semi-insulating (SI) GaAs (001) substrate followed by a 100-nm-thick undoped GaAs buffer layer, an 80-nm-thick undoped AlAs layer, a 1000 -nm-thick $n$-type $\quad\left(N_{\mathrm{Si}}=2 \times 10^{18} \mathrm{~cm}^{-3}, \quad n=2.3\right.$ $\times 10^{17} \mathrm{~cm}^{-3}$ ) $\quad \mathrm{Al}_{0.65} \mathrm{Ga}_{0.35} \mathrm{As}$ layer, and a 10 -nm-thick $n^{+}$GaAs cap, as illustrated in Fig. 1. The second sample was similar to the first except that it was $p$-doped and consisted of a SI GaAs (001) substrate followed by a 40-nm-thick undoped GaAs buffer, a 30-nm-thick undoped AlAs layer, a 1000 -nm-thick $\quad p$-type $\quad\left(N_{\mathrm{Be}}=2 \times 10^{18} \mathrm{~cm}^{-3}, \quad p=1.5\right.$ $\left.\times 10^{18} \mathrm{~cm}^{-3}\right) \quad \mathrm{Al}_{0.65} \mathrm{Ga}_{0.35} \mathrm{As}$ layer, and a 10 -nm-thick $p$ GaAs cap. The carrier densities of the $n$ - and $p$-type AlGaAs layers were determined using Hall effect measurements.

Samples suitable for observation by transmission electron microscopy (TEM) and electron holography were prepared using a Multiprep ${ }^{\mathrm{TM}}$ wedge-polishing apparatus

\begin{tabular}{|l|}
\hline $10 \mathrm{~nm}$ GaAs (n-doped) \\
\hline $1000 \mathrm{~nm} \mathrm{Al}_{0.65} \mathrm{Ga}_{0.35} \mathrm{As}$ (n-doped) \\
\hline 80nm AlAs \\
\hline $100 \mathrm{~nm} \mathrm{GaAs}$ buffer \\
\hline GaAs (001) substrate \\
\hline
\end{tabular}

FIG. 1. Schematic of $n$-doped heterostructure. 


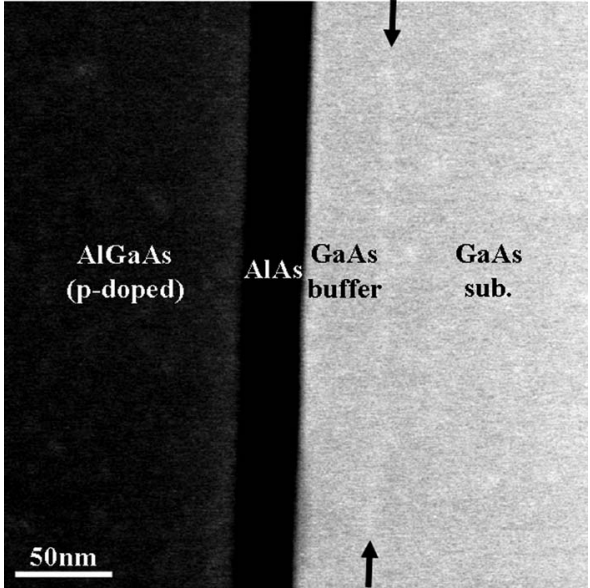

FIG. 2. Annular-dark-field scanning TEM image showing cross section of $p$-doped heterostructure. Note the faint line of brighter contrast indicated by arrows at buffer/substrate interface.

(wedge angle of $2^{\circ}$ ), followed by gentle argon ion milling for a few minutes at $3.5 \mathrm{keV}$, with a current of $13 \mu \mathrm{A}$ and a milling angle of $\pm 5^{\circ}$.

Off-axis electron holograms were recorded using a Philips CM200 FEG (field-emission gun) TEM operated at 200 $\mathrm{keV}{ }^{13}$ An electrostatic biprism was inserted in the selectedarea aperture plane, and a positive voltage was applied to the biprism. This arrangement caused the vacuum or reference wave to overlap with the object wave that passes through the sample. A typical biprism voltage of $130 \mathrm{~V}$ resulted in an interference fringe spacing of $5 \mathrm{~nm}$, and a typical primary image magnification of $15000 \times$ resulted in an effective

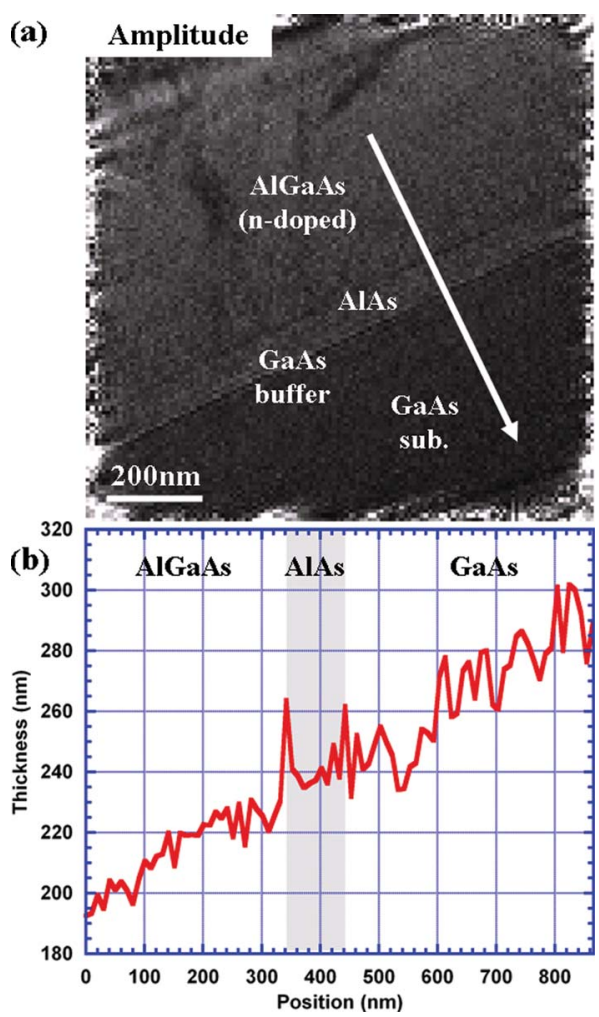

FIG. 3. (Color online) (a) Reconstructed amplitude image of $n$-doped heterostructure; (b) corresponding thickness line profile.

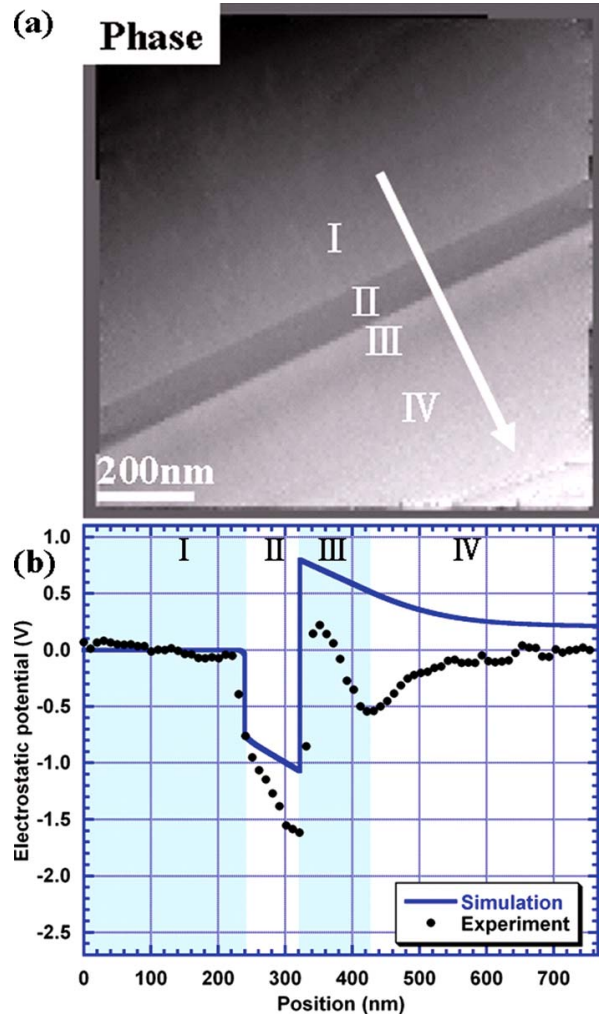

FIG. 4. (Color online) (a) Reconstructed phase image of $n$-doped heterostructure; (b) comparison of simulated and experimental potential profiles along line indicated in (a). Region I: $n$-doped AlGaAs; region II: AlAs; region III: GaAs buffer; and region IV: GaAs substrate.

pixel size of $10 \mathrm{~nm}$ in the reconstructed phase image. An additional weak Lorentz minilens located just beneath the bore of the lower objective-lens pole-piece was used to provide an enlarged field of view of $\sim 1 \mu \mathrm{m}$ for holographic analysis; as compared to a smaller field of view for imaging with the normal objective lens switched on. ${ }^{13}$ During the holography observations, the samples were typically tilted by $\sim 5^{\circ}$ away from the [110] zone axis about the substrate normal to minimize dynamical diffraction. ${ }^{14}$

\section{RESULTS AND DISCUSSION}

Figure 2 shows a typical cross section of the $p$-doped heterostructure recorded in the annular-dark-field imaging mode. The separate AlGaAs and AlAs layers are clearly visible. A faint line of brighter contrast can also be seen at the buffer/substrate interface, suggesting the presence of an unintentional delta-doped layer.

Figure 3(a) is an amplitude mage of the $n$-doped heterostructure, obtained after reconstruction of the off-axis electron hologram. A line profile was taken from the region indicated by the arrow in Fig. 3(a), taking into account the different values for the inelastic mean-free-path of GaAs (67 $\mathrm{nm}$ ), $\mathrm{AlAs}\left(77 \mathrm{~nm}\right.$ ), and $\mathrm{Al}_{0.65} \mathrm{Ga}_{0.35} \mathrm{As}$ (an interpolated value of $73 \mathrm{~nm}$ was used). ${ }^{15}$ The resulting thickness profile is shown in Fig. 3(b), and indicates a gentle thickness increase away from the specimen edge. This image confirms a welldefined specimen geometry and indicates minimum diffracting conditions within the analyzed area.

Figure 4(a) shows a reconstructed phase image of the 
$n$-doped heterostructure. The electrostatic potential profile is extracted from the thickness and phase images in Figs. 3(a) and 4(a) using the following expression: ${ }^{16}$

$$
\Phi=\frac{2 \pi e}{\lambda E} \times \frac{E_{0}+E}{2 E_{0}+E} V_{0} t=C_{E} V_{0} t
$$

where $e$ and $\lambda$ are the charge and wavelength of the incident electron, $E$ is the kinetic energy, $E_{0}$ is the rest energy, $C_{E}$ is an energy-related constant $\left(0.00728 \mathrm{rad} \mathrm{V}^{-1} \mathrm{~nm}^{-1}\right.$ for 200 $\mathrm{keV}$ electrons), and $V_{0}$ is the mean inner potential. The result is shown in Fig. 4(b) for the line profile indicated. The sample thickness $t$ in the analyzed area is estimated using the relationship between the incident electron-beam intensity and the reduced intensity caused by inelastic scattering. ${ }^{17}$

The electrostatic potential profile across the heterostructure is simulated using a 1D Poisson solver ${ }^{18}$ and compared to the experimental result in Fig. 4(b). The experimental measurements provide the electrostatic potential profile within an arbitrary voltage offset, which is chosen so that both the experimental and simulated potential profiles are zero in the AlGaAs layer. The values for the mean inner potential difference $\left(\Delta V_{0}\right)$ used in the simulation are (i) the published value of $1.9 \mathrm{~V}$ for the AlAs/GaAs interface $^{15}$ and (ii) an interpolated value of $0.7 \mathrm{~V}$ for the $\mathrm{Al}_{0.65} \mathrm{Ga}_{0.35} \mathrm{As} / \mathrm{Al}$ As interface, assuming a linear relationship between $\Delta V_{0}$ and composition. In comparing the experimental and simulated profiles, the change in the experimental potential is more than that predicted by the simulation in the AlAs layer. Most notably, the measured potential shows a substantial decrease in the GaAs buffer, a minimum at the buffer/substrate, and an increase in the substrate, which are not predicted by the simulation.

In order to improve the agreement with experiment, the simulation is revised to include a negative electrostatic sheet charge caused by unintentional impurities at the buffer/ substrate interface. The calculated band-edge energy diagram for the $n$-doped sample is shown in Fig. 5(a), where a $p$-type impurity sheet with an $8.4 \times 10^{11} \mathrm{~cm}^{-2}$ accepter concentration is included at the buffer/substrate interface. The electrostatic potentials for the experimental measurements and the simulations with and without the acceptor impurities are compared in Fig. 5(b). Further improvements between experiment and simulation are realized by adjusting $\Delta V_{0}$ on each side of the AlAs layer. The revised values are $0.87 \mathrm{~V}$ at the AlGaAs/AlAs interface and $1.85 \mathrm{~V}$ at the AlAs/GaAs interface, which are reasonable when uncertainties in the electrostatic potential measurements and the alloy composition are considered. Furthermore, both the experimental profile and the simulation with impurities show a potential decrease of $0.5 \mathrm{~V}$ at the buffer/substrate interface relative to the constant potential in the substrate, as indicated by the arrow. This potential drop corresponds to an electric field of 3.8 $\times 10^{4} \mathrm{~V} \mathrm{~cm}^{-1}$ and compares well with previously reported potential drops of $0.45 \mathrm{~V},{ }^{19}$ and electric fields on the order of $10^{4} \mathrm{~V} \mathrm{~cm}^{-1}$ in GaAs epilayer/GaAs substrate structures. ${ }^{20}$

The negative sheet charge can be explained by the presence of impurities on the surface of the substrate. Impurities such as carbon, silicon and oxygen at the epilayer/substrate interface have been reported in the literature, ${ }^{3,20}$ and the im-
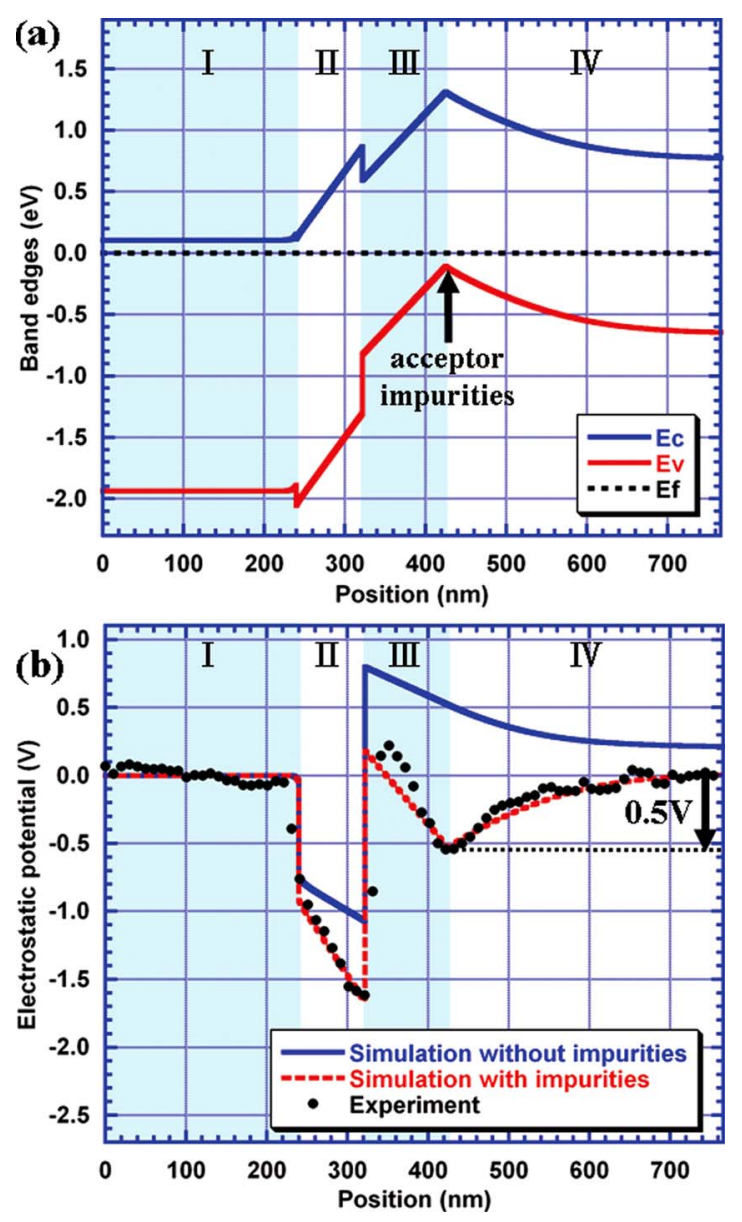

FIG. 5. (Color online) Band-edge energy diagram and electrostatic potential of $n$-doped heterostructure; region I: $n$-doped AlGaAs; region II: AlAs; region III: GaAs buffer; and region IV: GaAs substrate. (a) Band-edge energy simulation that includes a negative sheet charge due to impurities at buffer/ substrate interface. (b) Electrostatic potential comparisons between measurement and simulations with and without impurities at buffer/substrate interface.

purity concentrations have been reported to be growthcondition dependent ${ }^{8}$ and to range from a fraction to one monolayer. ${ }^{3}$ Carbon and silicon impurities can act as shallow acceptors and donors, respectively, for GaAs, while oxygen can be a deep-level impurity for nonradiative recombination centers. ${ }^{3,21}$ Reynolds and Geva ${ }^{10}$ have shown that there is a relationship between carrier depletion near the interface and net interface impurity $\left([\mathrm{C}]-[\mathrm{Si}] \mathrm{cm}^{-3}\right)$, suggesting that the amount of accumulated sheet charge is a function of $[\mathrm{C}]-[\mathrm{Si}] \mathrm{cm}^{-3}$ at the interface.

Similarly, the simulated and experimental electrostatic potentials are compared for the $p$-doped heterostructure. The band-edge energy diagram is shown in Fig. 6(a) and the electrostatic potential profile is shown in Fig. 6(b). The simulation with impurities again includes a sheet layer of 8.4 $\times 10^{11} \mathrm{~cm}^{-2}$ acceptors at the buffer/substrate interface. The values used for $\Delta V_{0}$ are $0.7 \mathrm{~V}$ at the AlGaAs/AlAs interface and $1.8 \mathrm{~V}$ at the $\mathrm{AlAs} / \mathrm{GaAs}$ interface. As before, the agreement with experiment is improved when the electrostatic potential simulation includes the presence of acceptor impurities at the buffer/substrate interface. Once again, the experiment and the simulation with impurities show a poten- 

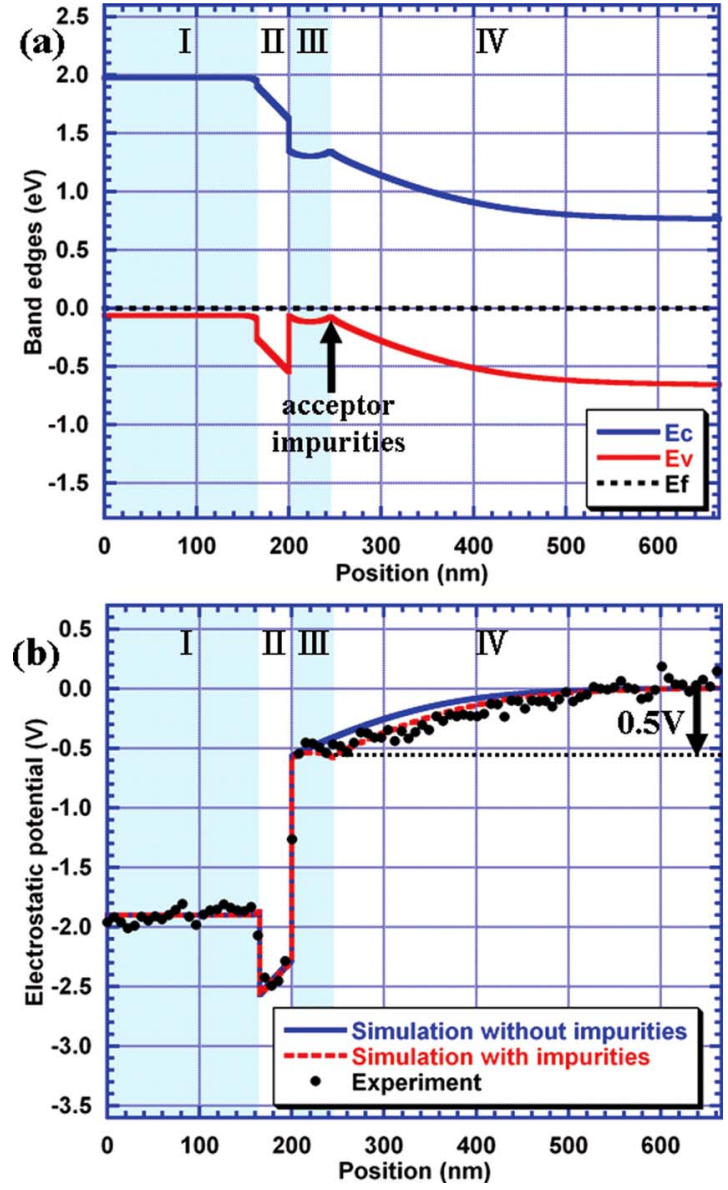

FIG. 6. (Color online) Band-edge energy diagram and electrostatic potential of $p$-doped heterostructure; region I: $p$-doped AlGaAs; region II: AlAs; region III: GaAs buffer; and region IV: GaAs substrate. (a) Band-edge energy simulation that includes a negative sheet charge due to impurities at buffer/ substrate interface. (b) Electrostatic potential comparisons between measurement and simulations with and without impurities at buffer/substrate interface.

tial decrease of $0.5 \mathrm{~V}$ at the buffer/substrate interface relative to the constant potential in the substrate, as indicated by the arrow.

\section{CONCLUSIONS}

Off-axis electron holography has been used to map the electrostatic potential profile across AlGaAs/AlAs/GaAs het- erostructures. Electrostatic potential drops were observed at the epilayer/substrate interface of both $n$ - and $p$-doped heterostructures and were attributed to ionized carbon acceptors. The measured potential profiles in the region of the buffer/ substrate interface were accurately simulated by adding an accepter impurity layer with a sheet concentration of 8.4 $\times 10^{11} \mathrm{~cm}^{-2}$ at the buffer/substrate interface. The holography technique could also be used to determine the sheet concentration of charge for the interface states at oxide/ semiconductor interfaces.

\section{ACKNOWLEDGMENTS}

This work was partially supported by the Department of Energy (Grant No. DE-FG02-04ER46168). We acknowledge the use of facilities in the John M. Cowley Center for High Resolution Electron Microscopy at Arizona State University.

${ }^{1}$ L. Esaki and R. Tsu, IBM Research Internal Report No. RC-2418, 1969.

${ }^{2}$ L. Esaki and R. Tsu, IBM J. Res. Dev. 14, 61 (1970).

${ }^{3}$ C. L. Reynolds, Jr., H. H. Vuong, and L. J. Peticolas, IEEE Trans. Electron Devices 39, 2459 (1992)

${ }^{4}$ M. L. Gray, C. L. Reynolds, and J. M. Parsey, Jr., J. Appl. Phys. 68, 169 (1990).

${ }^{5}$ H. Shen and M. Dutta, J. Appl. Phys. 78, 2151 (1995).

${ }^{6}$ H. Shen and F. H. Pollak, Phys. Rev. B 42, 7097 (1990).

${ }^{7}$ C. M. A. Kapteyn, F. Heinrichsdorff, O. Stier, R. Heitz, M. Grudmann, N. D. Zakharov, and D. Bimberg, Phys. Rev. B 60, 14265 (1999).

${ }^{8}$ M. Kaniewska and O. Engstrom, Mater. Sci. Eng., C 27, 1069 (2007).

${ }^{9}$ J. M. Ortion, Y. Cordier, J. C. Garcia, D. Adam, and C. Grattepain, Jpn. J. Appl. Phys., Part 1 35, 5964 (1996).

${ }^{10}$ C. L. Reynolds, Jr. and M. Geva, J. Appl. Phys. 72, 303 (1992).

${ }^{11}$ M. R. McCartney and D. J. Smith, Annu. Rev. Mater. Sci. 37, 729 (2007).

${ }^{12}$ M. R. McCartney, M. A. Gribelyuk, J. Li, P. Ronsheim, J. S. McMurray, and D. J. Smith, Appl. Phys. Lett. 80, 3213 (2002).

${ }^{13}$ M. R. McCartney, D. J. Smith, R. F. C. Farrow, and R. J. Marks, J. Appl. Phys. 82, 2461 (1997).

${ }^{14}$ M. Gajdardziska-Josifovska, M. R. McCartney, W. J. de Ruijter, D. J. Smith, J. K. Weiss, and J. M. Zuo, Ultramicroscopy 50, 285 (1993).

${ }^{15}$ S. Chung, D. J. Smith, and M. R. McCartney, Microsc. Microanal. 13, 329 (2007).

${ }^{16}$ L. Reimer, Transmission Electron Microscopy (Springer, Berlin, 1991)

${ }^{17}$ M. R. McCartney and M. Gajdardziska-Josifovska, Ultramicroscopy $\mathbf{5 3}$, 283 (1994).

${ }^{18}$ G. Snider, 1D Poisson program, available at http://www.nd.edu/ gsnider/.

${ }^{19}$ C. Heinlein, J. K. Grepstad, B. O. Fimland, and T. Berge, J. Cryst. Growth 201-202, 586 (1999)

${ }^{20}$ J. Luyo-Alvarado, M. Melendez-Lira, M. Lopez-Lopeza, and S. Goto, J. Vac. Sci. Technol. B 19, 495 (2001).

${ }^{21}$ K. Akimoto, M. Kamada, K. Taira, M. Arai, and N. Watanabe, J. Appl. Phys. 59, 2833 (1986). 